\title{
Left Ventricular global longitudinal strain predicts heart failure readmission in acute decompensated heart failure
}

\author{
Simone Romano ${ }^{1,2}$, Ibrahim N. Mansour ${ }^{1}$, Mayank Kansal ${ }^{1}$, Hana Gheith ${ }^{1}$, Zachary Dowdy ${ }^{1}$, Carolyn A. Dickens ${ }^{1}$, \\ Cassandra Buto-Colletti ${ }^{1}$, June M. Chae ${ }^{1}$, Hussam H. Saleh ${ }^{1}$ and Thomas D. Stamos ${ }^{1 *}$
}

\begin{abstract}
Background: The goal of this study was to determine if left ventricular (LV) global longitudinal strain (GLS) predicts heart failure (HF) readmission in patients with acute decompensated heart failure.

Methods and results: Two hundred ninety one patients were enrolled the time of admission for acute decompensated heart failure between January 2011 and September 2013. Left ventricle global longitudinal strain (LV GLS) by velocity vector imaging averaged from 2, 3 and 4-chamber views could be assessed in 204 out of 291 (70\%) patients. Mean age was $63.8 \pm 15.2$ years, $42 \%$ of the patients were males and $78 \%$ were African American or Hispanic. Patients were followed until the first HF hospital readmission up to 44 months. Patients were grouped into quartiles on the basis of LV GLS. Kaplan-Meier curves showed significantly higher readmission rates in patients with worse LV GLS (log-rank $p<0.001$ ). After adjusting for age, sex, history of ischemic heart disease, dementia, New York Heart Association class, LV ejection fraction, use of angiotensin converting enzyme inhibitors or angiotensin receptor blockers, systolic and diastolic blood pressure on admission and sodium level on admission, worse LV GLS was the strongest predictor of recurrent HF readmission $(p<0.001)$. The ejection fraction was predictive of readmission in univariate, but not in multivariate analysis.
\end{abstract}

Conclusion: LV GLS is an independent predictor of HF readmission after acute decompensated heart failure with a higher risk of readmission in case of progressive worsening of LV GLS, independent of the ejection fraction.

Keywords: Heart failure, Echocardiography, Strain analysis

\section{Background}

Left ventricular ejection fraction (LVEF) is the most commonly used parameter of systolic function [1]. It is essential for the management of heart failure (HF) patients, particularly to guide therapy and for prognostication $[2,3]$. Recently there has been great interest in development of novel quantitative methods to assess systolic function $[4,5]$. One promising technique is two-dimensional speckle tracking which can provide information on the rate of segmental and global myocardial deformation. Global Longitudinal Strain (GLS) is defined as the change of length of a tissue normalized to its original length $\left(\left[\mathrm{L}-\mathrm{L}_{0}\right] / \mathrm{L}_{0}\right)$.

\footnotetext{
* Correspondence: tstamos@uic.edu

${ }^{1}$ Division of Cardiology, Department of Medicine, University of Illinois at

Chicago, 840 S. Wood Street, M/C 715, Chicago, IL 60612, USA

Full list of author information is available at the end of the article
}

Prior studies have reported the usefulness of GLS for prognostic stratification of HF outpatients [6-9], however only one previous study found this parameter to be predictive in patients admitted to the hospital with acute decompensated heart failure (ADHF) in a mostly white European patient population (98\%) [10].

We hypothesized that GLS could be a useful predictor of readmission in a mostly African American patient population hospitalized with ADHF.

\begin{abstract}
Methods
This was a single-center retrospective observational study that involved chart and medical record reviews, and analysis of de-identified clinical data and previously recorded echocardiographic tracings. Chart review was performed on 291 patients, aged $\geq 18$ years, who were
\end{abstract}


admitted to the University of Illinois Hospital and Health Sciences System (UI-Health) with the primary admission diagnosis of ADHF from January 2011 thru September 2013.

Inclusion criteria consisted of patients aged 18 years or older who had been admitted between January 2011 and September 2013 at the University of Illinois Hospital and Health Sciences System with the diagnosis of heart failure, either as new diagnosis or as acute decompensation of chronic HF, had a complete Transthoracic Echocardiography, and did not meet any of the exclusion criteria.

Exclusion criteria consisted of age under 18 years, diagnosis of terminal cancer, diagnosis of diseases, other than heart failure that could cause volume overload, such as end-stage renal disease requiring hemodialysis, severe liver disease, pericardial tamponade or constriction, acute myocardial infarction, and primary valvular disease, diagnosis of acute coronary syndrome, procedures that might affect prognosis (cardiac bypass surgery, coronary artery stenting), and lack of contact with the hospital. The diagnosis of ADHF was confirmed if the admitting cardiologist included heart failure as the primary admitting diagnosis and the documented physical examination laboratory and radiologic findings were consistent with this diagnosis. Following the index HF admission the medical record was assessed to determine hospital readmission, with a follow-up period of up to 44 months. Patients with a non-cardiovascular death prior to HF readmission were censored. Data was obtained from the comprehensive review of the medical record and cardiologist's admission note regarding medical history and physical examination. The baseline characteristics of patients were collected upon hospital admission except the New York Heart functional class (NYHA) that was obtained from the most recent clinic visit when the patient was clinically stable prior to the index admission. When admitted, all patients were in NYHA class III or IV.

\section{Transthoracic echocardiography and global longitudinal strain}

Standard 4-chamber, 3-chamber and 2-chamber apical views and parasternal short-axis views of the left ventricle were obtained using a commercially available ultrasound system. All images were stored digitally and analyzed with offline software (Syngo Dynamics 9.0 software, Siemens Medical Solutions). The majority of echocardiography studies were performed within $24 \mathrm{~h}$ after admission, with all studies being completed by $48 \mathrm{~h}$ post-admission. We used these images to calculate GLS and EF that were included in the analysis.

Speckle tracking for myocardial strain was performed using Velocity Vector Imaging software (Siemens
Medical Systems, Erlangen, Germany). A digital loop was acquired from apical 2-3 and 4 chamber views. The GLS was the average result of three measurements for each view. The software calculated the endocardial average strain values from 6 left ventricle segments for a total of 18 segments, therefore the GLS was the result of the average of 18 segments. We obtained GLS only in the case of adequate tracking quality at least in 5 of the 6 segments per view. LV ejection fraction was averaged from the three apical views by automated endocardial tracking of end-diastolic and end-systolic volumes. All measurements were made blinded to other results and clinical details.

\section{Adequacy of measurements}

Multiple studies have demonstrated the low measurement variability for GLS [6-10]. In our software, left ventricle endocardial borders were manually traced at the end-diastole in ECG-gated long axis views. Subsequently, the software's automatic border tracking algorithm, which tracks image features throughout the whole cardiac cycle, was applied. Accurate tracking was ascertained by visual assessment of all borders. Images with inadequate tracking of the endocardial were excluded from the analysis.

\section{Outcome}

Readmission to UI-Health for HF following the index ADHF admission was assessed using the electronic medical record.

\section{Statistical analysis}

The number of patients and percentages were calculated for categorical variables. Means, standard deviations, and medians were calculated for continuous variables. Receiver operating characteristic (ROC) curve was used to assess the optimal GLS threshold value which maximized the average of sensitivity and specificity for predicting HF admission. The area under the curve, which was a measure of the discriminatory power of the predictor, ranged from 0.5 to 1 . Clinical characteristics were compared among patients categorized by quartiles of GLS, using the Chi-square test for categorical variables and $t$-test for continuous variables. Univariate and multivariate cox proportional hazard models were used to examine the association of GLS and HF readmission. Variables significantly correlated with outcomes on univariate analysis $(p<0.05)$ or known to influence outcomes were incorporated into the multivariate analyses. Accordingly, hazard ratios (HR) and 95\% confidence interval (CI) were calculated. The Kaplan-Meier curves and log-rank tests were used to compare the time to first heart failure readmission across quartiles of GLS. 
Statistical analyses were conducted using IBM SPSS 21 (Armonk, NY). The significance level was set at 0.05.

\section{Results}

\section{Population characteristics}

In 204 (70\%) patients, LV GLS by velocity vector imaging of the 2, 3 and 4-chamber views could be assessed. As shown in Table 1 , mean age was $63.8 \pm 15.2$ years, $42 \%$ were male, and ethnicity distribution was $71 \%$ African Americans, 7\% Caucasian and 7\% Hispanics, the remaining $15 \%$ a mixture of other minorities. 49 patients had heart failure with preserved ejection fraction (HFpEF) (EF > 50\%). The average LVEF was $40 \%$ and the average NYHA functional class during the clinic visit prior to their index hospitalization was 2.03. None of the patients was treated with coronary revascularization, implantable cardioverter defibrillator or cardiac resynchronization therapy after index hospitalization.

The area under the ROC curve for prediction of HF readmission using LV GLS was 0.783 (Fig. 1). Patients were grouped into quartiles according to LV GLS as follows, $<-14.15 \%,-14.15 \%$ to $-10.55 \%,-10.54 \%$ to $-6.41 \%$ and $>-6.41 \%$ demonstrating increased readmission with worse strain quartiles. Baseline characteristics of patients with a GLS above and below the threshold value are summarized in Table 1.

\section{Readmission}

A total of 113 patients (55\%) had at least one readmission. In the first quartile there were $10(9 \%)$ patients with at least one readmission, 24 patients $(21 \%)$ in the second quartile, 38 patients (34\%) in the third quartile and 41 patients (36\%) in the fourth quartile. KaplanMeier curves (Fig. 2) demonstrated significantly higher admission rate in the more altered GLS group $(p<$ 0.001). On univariate Cox proportional hazard analyses (Table 2), more altered GLS was significantly associated with $\mathrm{HF}$ admissions, either as continuous variable (HR 1.17, CI 1.12-1.23, $p<0.001$ ) or with lower quartiles of GLS -14.15 to -10.55 (HR 3.1, CI 1.46-6.74, p 0.003),

Table 1 Baseline characteristics of the entire cohort and by quartiles of GLS

\begin{tabular}{|c|c|c|c|c|c|c|}
\hline \multirow[t]{2}{*}{ Characteristic } & \multirow{2}{*}{$\begin{array}{l}\text { Whole } \\
\text { population }\end{array}$} & \multicolumn{4}{|l|}{ LV GLS } & \multirow[t]{2}{*}{$P$ Value } \\
\hline & & $<-14.15$ & -14.5 to -10.55 & -10.54 to -6.41 & $>-6.41$ & \\
\hline Age (years) & $63.8 \pm 15$ & $66.3 \pm 14$ & $63.4 \pm 15.8$ & $65.7 \pm 13.9$ & $59.7 \pm 16.3$ & 0.116 \\
\hline Male & 87 (42.4\%) & $11(21.6 \%)$ & $19(37.3 \%)$ & $25(48.1 \%)$ & $32(62.7 \%)$ & $<0.001$ \\
\hline Sodium level & $138.3 \pm 3.4$ & $138.1 \pm 4.2$ & $139 \pm 3$ & $138.7 \pm 3.4$ & $137.5 \pm 2.9$ & 0.153 \\
\hline Creatinine & $2.2 \pm 2.4$ & $2.6 \pm 3.6$ & $2.6 \pm 2.5$ & $1.7 \pm 1.2$ & $1.8 \pm 1.5$ & 0.163 \\
\hline LVEF & $40.4 \pm 17.4$ & $60.4 \pm 7.5$ & $47.8 \pm 11.6$ & $30.9 \pm 10.4$ & $22.3 \pm 5.9$ & $<0.001$ \\
\hline \multicolumn{7}{|l|}{ Medication use } \\
\hline ACEI or ARBs & $140(69.7 \%)$ & $25(49 \%)$ & $32(65.3 \%)$ & 39 (78\%) & 44 (86.3\%) & $<0.001$ \\
\hline$\beta$-Blocker & $173(86.1 \%)$ & $37(72.5 \%)$ & $43(87.8 \%)$ & 47 (94\%) & 46 (90.2\%) & 0.011 \\
\hline Hydralazine & $55(27.5 \%$ & $14(27.5 \%)$ & $21(43.8 \%)$ & $9(18 \%)$ & $11(21.6 \%)$ & 0.023 \\
\hline Ca channel Blockers & $45(22.6 \%)$ & $17(33.3 \%)$ & 19 (39.6\%) & $6(12 \%)$ & $3(6 \%)$ & $<0.001$ \\
\hline Loop diuretics & $164(81.6 \%)$ & $41(80.4 \%)$ & 37 (75.5\%) & $41(82 \%)$ & $45(88.2 \%)$ & 0.43 \\
\hline Aldosterone-antagonists & $19(9.7 \%)$ & $1(2 \%)$ & $3(6.3 \%)$ & $5(10.4 \%)$ & $10(19.6 \%)$ & 0.021 \\
\hline \multicolumn{7}{|l|}{ NYHA functional class } \\
\hline Class 1 & $55(28.8 \%)$ & $17(36.2 \%)$ & $16(34 \%)$ & $14(28.6 \%)$ & $8(16.7 \%)$ & \\
\hline Class II & $78(40.8 \%)$ & 19 (40.4\%) & 15 (31.9\%) & $24(49 \%)$ & $20(41.7 \%)$ & 0.087 \\
\hline Class III & $55(28.8 \%)$ & $9(19.1 \%)$ & $16(34 \%)$ & $10(20.4 \%)$ & $20(41.7 \%)$ & \\
\hline Class IV & $3(1.6 \%)$ & $2(4.3 \%)$ & 0 & $1(2 \%)$ & 0 & \\
\hline \multicolumn{7}{|l|}{ Blood pressure (mmHg) } \\
\hline Systolic & $148.7 \pm 38.4$ & $158.5 \pm 35.9$ & $166.5 \pm 38$ & $145.2 \pm 36.6$ & $124.7 \pm 29.7$ & $<0.001$ \\
\hline Diastolic & $84.9 \pm 21.6$ & $83.1 \pm 23.5$ & $89.1 \pm 22.2$ & $86.1 \pm 21.9$ & $81.3 \pm 18.2$ & 0.28 \\
\hline Heart rate (bpm) & $86.1 \pm 21.5$ & $79.2 \pm 19.1$ & $86 \pm 20.3$ & $87.7 \pm 22.2$ & $91.6 \pm 23.1$ & 0.032 \\
\hline History of ischemic heart disease & $80(39 \%)$ & $13(25.5 \%)$ & $23(45.1 \%)$ & 27 (51.9\%) & $17(33.3 \%)$ & 0.029 \\
\hline Chronic obstructive pulmonary disease & $30(14.6 \%)$ & $6(11.8 \%)$ & $10(19.6 \%)$ & $6(11.5 \%)$ & $8(15.7 \%)$ & 0.617 \\
\hline Diabetes & 79 (38.5\%) & $15(29.4 \%)$ & $23(45.1 \%)$ & $24(46.2 \%)$ & $17(33.3 \%)$ & 0.206 \\
\hline Hypertension & $190(93.1 \%)$ & $46(90.2 \%)$ & $50(98 \%)$ & 49 (94.2\%) & $45(90 \%)$ & 0.324 \\
\hline
\end{tabular}




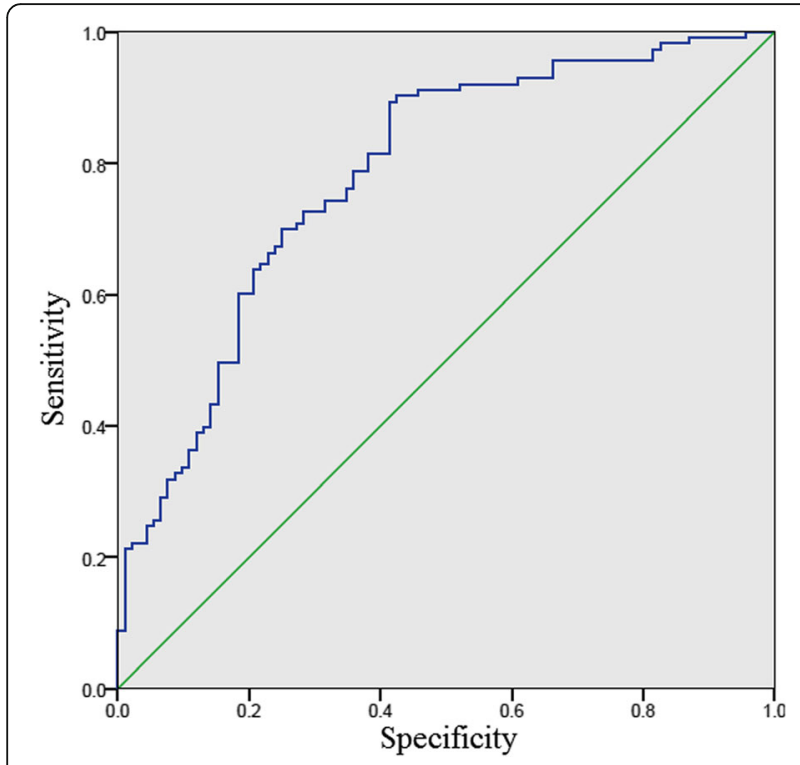

Fig. 1 ROC curve. The area under the ROC curve for prediction of HF readmission using LV GLS was $0.783(p<0.001)$

-10.54 and $-6.41 \%$ (HR 7.4, CI 3.55-15.39, $p<0.001$ ), and $>-6.41 \% \quad(\mathrm{HR} \quad 7.8, \quad \mathrm{CI}$ 3.79-16.34, $p<0.001)$. Additional variables significantly associated with HF admission in univariate analysis included LVEF ( $p$ $<0.001$ ), NYHA functional class III (p 0.01), systolic blood pressure $(p<0.001)$, diastolic blood pressure $(p$ $0.025)$, history of ischemic heart disease ( $p$ 0.021), dementia ( $p$ 0.018) sodium level ( $p$ 0.018) and angiotensin converting enzyme inhibitors-angiotensin receptor blockers (ACEI-ARBs) use ( $p$ 0.008). In the multivariate analysis (Table 3), after adjusting for age, sex, history of ischemic heart disease, dementia, NYHA class, LV
Table 2 Univariate analyses of factors that were shown to be significantly correlated to HF readmissions

\begin{tabular}{llll}
\hline HF Readmission & & & \\
\hline Variable & Hazard ratio & $95 \% \mathrm{Cl}$ & $p$-value \\
LV GLS -14.15 to -10.55 & 3.144 & $1.46-6.74$ & 0.003 \\
LV GLS -10.54 to 6.41 & 7.40 & $3.55-15.39$ & $<0.001$ \\
LV GLS $>-6.41$ & 7.87 & $3.79-16.34$ & $<0.001$ \\
LV GLS as a continuous variable ${ }^{a}$ & 1.17 & $1.12-1.23$ & $<0.001$ \\
LVEF & 0.97 & $0.96-0.98$ & $<0.001$ \\
Age & 1.01 & $0.99-1.02$ & 0.099 \\
Male & 1.11 & $0.77-1.61$ & 0.566 \\
NYHA functional class II & 1.3 & $0.77-2.19$ & 0.314 \\
NYHA functional class III & 2.01 & $1.18-3.41$ & 0.01 \\
NYHA functional class IV & 0.92 & $0.12-6.87$ & 0.937 \\
Systolic blood pressure & 0.98 & $0.98-0.99$ & $<0.001$ \\
Diastolic blood pressure & 0.99 & $0.98-0.99$ & 0.025 \\
Heart rate & 1 & $0.99-1.01$ & 0.137 \\
Creatinine & 0.9 & $0.82-1$ & 0.052 \\
Sodium level & 0.93 & $0.88-0.98$ & 0.018 \\
History of ischemic heart disease & 1.55 & $1.07-2.24$ & 0.021 \\
Diabetes & 1 & $0.68-1.46$ & 0.983 \\
Hypertension & 1.45 & $0.59-3.55$ & 0.418 \\
Dementia & 12.18 & $1.54-95.95$ & 0.018 \\
Chronic obstructive pulmonary & 1.24 & $0.76-2.01$ & 0.382 \\
disease & & $1.17-2.86$ & 0.008 \\
ACEI-ARBs & 1.83 & & \\
\hline A differt & & & \\
\hline
\end{tabular}

${ }^{\mathrm{a} A}$ different model with the same variables was performed using LV GLS as a continuous variable

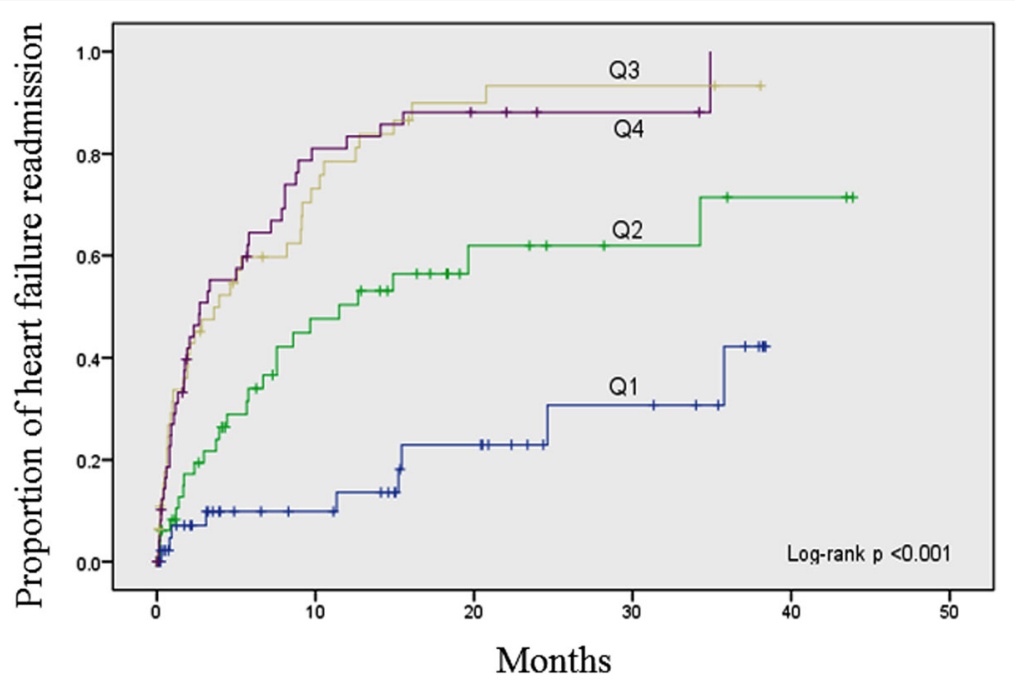

Fig. 2 Kaplan-Meier curves. Kaplan-Meier curves showing higher heart failure readmissions in patients within worse LV GLS quartiles (Q). Q1 <-14.15, Q2 -14.15 to 10.55, Q3 -10.54 to -6.41, Q4 > - 6.41 
Table 3 Multivariate Cox regression analysis incorporating factors that were shown to be significantly correlated with HF readmissions in univariate analyses

\begin{tabular}{|c|c|c|c|c|}
\hline Variable & Number/Mean/Percentage & Hazard Ratio & 95\% Confidence Interval & $p$-value \\
\hline LV GLS Quartile 2 (-14.15 to -10.55$)$ & $N=51$ & 3.16 & $1.26-7.90$ & 0.014 \\
\hline LV GLS Quartile 3 (-10.54 to -6.41$)$ & $N=52$ & 5.19 & $1.70-15.82$ & 0.004 \\
\hline LV GLS Quartile 4 (>-6.41) & $N=51$ & 5.30 & $1.43-19.60$ & 0.012 \\
\hline LV GLS as a continuous variable ${ }^{a}$ & $-10.6 \pm 4.7 \%$ & 1.23 & $1.09-1.40$ & 0.001 \\
\hline Age & $63.8 \pm 15$ & 1.02 & $1.002-1.03$ & 0.027 \\
\hline Left ventricular ejection fraction & $40 \pm 17 \%$ & 0.98 & $0.95-1.02$ & 0.288 \\
\hline History of ischemic heart disease & $39 \%$ & 1.13 & $0.71-1.78$ & 0.608 \\
\hline NYHA III & $29 \%$ & 1.79 & $0.98-3.29$ & 0.060 \\
\hline Use of ACEI/ARBs & $70 \%$ & 0.96 & $0.565-1.65$ & 0.894 \\
\hline Systolic blood pressure & $149 \pm 38$ & 0.999 & $0.99-1.01$ & 0.689 \\
\hline Sodium level & $138 \pm 3$ & 0.94 & $0.88-1.00$ & 0.53 \\
\hline
\end{tabular}

${ }^{\mathrm{a}} \mathrm{A}$ different model with the same variables was performed using LV GLS as a continuous variable

ejection fraction, use of ACEI or ARBs, systolic and diastolic blood pressure on admission and sodium level on admission, worse LV GLS was the strongest predictor of recurrent $\mathrm{HF}$ readmission either as continuous variable (HR 1.23, CI 1.09-1.4, $p=0.001$ ) or using the cut-off point of -14.15 to -10.55 (HR 3.6, CI 1.26-7.9, p 0.014), between -10.54 and $-6.41 \%$ (HR 5.19, CI 1.7-15.82, $p$ $<0.001$ ), and $>-6.41 \%$ (HR 5.3, CI 1.43-19.6, $p<0.001$ ). Ejection fraction was a univariate predictor for readmission, but not a multivariate predictor. The small number of patients with preserved LVEF precluded a useful analysis of this subgroup.

A dedicated statistical analysis to evaluate the correlation between GLS and all-cause readmission did not demonstrate a statistically significant correlation (data not shown).

\section{Discussion}

In this study, we demonstrate that after adjusting for factors that can affect clinical outcomes, LV GLS is a strong and independent predictor of HF readmission following an index admission for ADHF. This is the first study to show GLS can predict readmission in a racially diverse group of patients with ADHF.

We know of only two other studies to assess strain in patients with ADHF. One of the previously published studies differed from our study in several important ways [11]. Whereas our study included patients with both heart failure with reduced ejection fraction (HFrEF) and HFpEF, the previous study appears to have focused on HFrEF. Furthermore, they found global circumferential strain (GCS) to predict outcome and not GLS. Our study found GLS to be an independent predictor. GLS is thought to be an early marker of subclinical LV dysfunction, whereas GCS becomes abnormal later in the course of myocardial disease [12]. It is unclear why they did not find GLS to be predictive, as one would expect this parameter to become abnormal before GCS. One might hypothesize that GLS would be a better predictor than GCS in a lower risk group due to its ability to detect myocardial dysfunction at an earlier stage.

The only other study to demonstrate GLS as a predictor of readmission [10] had a population consisting of 98\% white Europeans and their follow-up was only 30 days. This differs from our study which included a largely African American patient population. This is an important discovery considering data exists to suggest African American patients with heart failure remodel differently than whites, raising the possibility that GLS would predict differently in this patient population [13].

Despite dramatic improvement in outcomes with medical therapy for heart failure, readmission rates remain high, with approximately $50 \%$ of HF patients rehospitalized within 6 months of discharge [14]. Although studies have found factors that can predict readmission in large administrative databases, assembling a risk model that can reliably predict readmission in individual patients has been less than successful $[15,16]$. The ability to identify a group at high risk for readmission using GLS might allow for resources to be directed towards these individuals in order to reduce the readmission rate.

\section{Limitations}

This was a single center study with information being obtained in a retrospective manner. The clinical use of GLS is limited considering its feasibility in only $70 \%$ of cases in our study. Also GLS evaluation by echocardiography requires good quality images in order to obtain reliable measurements. Two dimensional and Doppler echocardiographic parameters were not included in the present study. 


\section{Conclusion}

Our study is the first to demonstrate GLS as a predictor of readmission in a largely minority patient population with ADHF, which was independent of LVEF and other known clinical risk predictors. Further study to determine if GLS can impact outcomes in these patients is warranted.

\section{Abbreviations}

ACEl: Angiotensin converting enzyme inhibitors; ADHF: Acute decompensated heart failure; ARBs: Angiotensin receptor blockers; Cl: Confidence interval; GCS: Global circumferential strain; GLS: Global Longitudinal Strain; HF: Heart Failure; HFpEF: Heart failure with preserved ejection fraction; HFrEF: Heart failure with reduced ejection fraction; HR: Hazard ratio; LV: Left ventricle; LVEF: Left ventricular ejection fraction; NYHA: New York Heart Association; ROC: Receiver operating characteristic; Ul-Health: University of Illinois Hospital and Health Sciences System

\section{Acknowledgements}

Not applicable.

\section{Funding}

None.

\section{Availability of data and materials}

the datasets used and/or analysed during the current study available from the corresponding author on reasonable request.

\section{Authors' contributions}

SR: Data collection, Data analysis, drafting article. IM: Statistics. MK: Critical revision of the article, approval. $H G, Z D, C D, C B, J C, H S$ : Data collection. TS: Concept/design, Critical revision of the article, approval. All authors read and approved the final manuscript.

\section{Competing interests}

The authors declare that they have no competing interests.

\section{Consent for publication}

Not applicable.

\section{Ethics approval and consent to participate}

Not applicable.

\section{Publisher's Note}

Springer Nature remains neutral with regard to jurisdictional claims in published maps and institutional affiliations.

\section{Author details}

${ }^{1}$ Division of Cardiology, Department of Medicine, University of Illinois at Chicago, 840 S. Wood Street, M/C 715, Chicago, IL 60612, USA. ²Department of Medicine, University of Verona, Ospedale policlinico Borgo Roma, Piazzale scuro 10, 37134 Verona, Italy.

Received: 7 November 2016 Accepted: 1 March 2017

Published online: 15 March 2017

\section{References}

1. Dickstein K, Cohen-Solal A, Filippatos G, McMurray JJ, Ponikowski P, Poole-Wilson PA, Strömberg A, Van Veldhuisen DJ, Atar D, Hoes AW, Keren A, Mebazaa A, Nieminem M, Priori SG, Swedberg K. ESC committee for practice guidelines (CPG). guidelines for the diagnosis and treatment of acute and chronic heart failure 2008: the task force for the diagnosis and treatment of acute and chronic heart failure 2008 of the european society of cardiology. Eur Heart J. 2008:29:2388-442.

2. Solomon SD, Anavekar N, Skali H, McMurray JJ, Swedberg K, Yusuf S, Granger CB, Michelson EL, Wang D, Pocock S, MA P. Candesartan in heart failure reduction in mortality (CHARM) investigators. Influence of ejection fraction on cardiovascular outcomes in a broad spectrum of heart failure patients. Circulation. 2005;112:3738-44.
3. Vardas PE, Auricchio A, Blanc JJ, Daubert JC, Drexler H, Ector H, Gasparini M, Linde C, Morgado FB, Oto A, Sutton R, Trusz-Gluza M. European society of cardiology; european heart rhythm association. Guidelines for cardiac pacing and cardiac resynchronization therapy. The task force for cardiac pacing and cardiac resynchronization therapy of the european society of cardiology. Developed in collaboration with the european heart rhythm association. Europace. 2007;9:959-98.

4. Marwick TH. Methods used for the assessment of LV systolic function: common currency or tower of babel? Heart. 2013;99:1078-86.

5. Thomas JD, Popovic ZB. Assessment of left ventricular function by cardiac ultrasound. J Am Coll Cardiol. 2006:48:2012-25.

6. Mignot A, Donal E, Zaroui A, Reant P, Salem A, Hamon C, Monzy S, Roudaut $\mathrm{R}$, Habib G, Lafitte S. Global longitudinal strain as a major predictor of cardiac events in patients with depressed left ventricular function: a multicenter study. J Am Soc Echocardiogr. 2010;23:1019-24.

7. Stanton T, Leano R, Marwick TH. Prediction of All-cause mortality from global longitudinal speckle strain. Comparison with ejection fraction and wall motion scoring. Circ Cardiovasc Imaging. 2009;2:356-64.

8. Motoki H, Borowski AG, Shrestha K, Troughton RW, Tang WH, Thomas JD, Klein AL. Incremental prognostic value of assessing left ventricular myocardial mechanics in patients with chronic systolic heart failure. JACC. 2012;60:2074-81.

9. Zhang KW, French B, May Khan A, Plappert T, Fang JC, Sweitzer NK, Borlaug BA, Chirinos JA, St John Sutton M, Cappola TP, Ky B. Strain improves risk prediction beyond ejection fraction in chronic systolic heart failure. J Am Heart Assoc. 2014;3(1):e000550. doi:10.1161/JAHA.113.000550.

10. Saito M, Negishi K, Eskandari M, Huynh Q, Hawson J, Moore A, Koneru S, Foster S, Marwick TH. Association of left ventricular strain with 30-Day mortality and readmission in patients with heart failure. J Am Soc Echocardiogr. 2015;28:652-66.

11. Cho GY, Marwick TH, Kim HS, Kim MK, Hong KS, Oh DJ. Global 2 dimensional strain as a new prognosticator in patients with heart failure. JAAC. 2009;54(7):618-24. doi:10.1016/j.jacc.2009.04.061.

12. Mor-Avi V, Lang RM, Badano LP, Belohlavek M, Cardim NM, Derumeaux G, Galderisi M, Marwick T, Nagueh SF, Sengupta PP, Sicari R, Smiseth OA, Smulevitz B, Takeuchi M, Thomas JD, Vannan M, Voigt JU, Zamorano JL. Current and evolving echocardiographic techniques for the quantitative evaluation of cardiacmechanics: ASE/EAE consensus statement on methodology and indications endorsed by the japanese society of echocardiography. J Am Soc Echocardiogr. 2011;24(3):277-313. doi:10.1016/ j.echo.2011.01.015.

13. Okin PM, Kjeldsen SE, Dahlöf B, Devereux RB. Racial differences in incident heart failure during antihypertensive therapy. Circ Cardiovasc Qual Outcomes. 2011:4(2):157-64.

14. Butler J, Kalogeropoulos A. Worsening heart failure hospitalization epidemic we do not know how to prevent and we do not know how to treat! J Am Coll Cardiol. 2008;52(6):435-7. doi:10.1016/j.jacc.2008.04.037.

15. Kansagara D, Englander H, Salanitro A, Kagen D, Theobald C, Freeman M, Kripalani S. Risk prediction models for hospital readmission: a systematic review. JAMA. 2011;306:1688-98.

16. Ross JS, Mulvey GK, Stauffer B, Patlolla V, Bernheim SM, Keenan PS, Krumholz HM. Statistical models and patient predictors of readmission for heart failure: a systematic review. Arch Intern Med. 2008;168:1371-86.

Submit your next manuscript to BioMed Central and we will help you at every step:

- We accept pre-submission inquiries

- Our selector tool helps you to find the most relevant journal

- We provide round the clock customer support

- Convenient online submission

- Thorough peer review

- Inclusion in PubMed and all major indexing services

- Maximum visibility for your research

Submit your manuscript at www.biomedcentral.com/submit
Ciomed Central 